\title{
Photoluminescence spectroscopy of nearly defect-free InN microcrystals exhibiting nondegenerate semiconductor behaviors
}

\author{
Ching-Lien Hsiao, Hsu-Cheng Hsu, and Li-Chyong Chen ${ }^{\text {a) }}$ \\ Center for Condensed Matter Sciences, National Taiwan University, Taipei 106, Taiwan \\ Chien-Ting Wu and Chun-Wei Chen \\ Materials Science and Engineering, National Taiwan University, Taipei 106, Taiwan \\ Min Chen and Li-Wei Tu \\ Department of Physics and Center for Nanoscience and Nanotechnology, National Sun Yat-Sen University, \\ Kaohsiung 80424, Taiwan \\ Kuei-Hsien Chen \\ Institute of Atomic and Molecular Sciences, Academia Sinica, Taipei 106, Taiwan
}

(Received 12 July 2007; accepted 11 October 2007; published online 1 November 2007)

\begin{abstract}
Nearly defect-free InN microcrystals grown on $\mathrm{Si}(111)$ substrates have been realized by plasma-assisted molecular beam epitaxy. High-resolution transmission electron microscope images reveal that these microcrystals exhibit single-crystalline wurtzite structure. Low temperature photoluminescence (PL) shows a strong emission peak at $0.679 \mathrm{eV}$ with a very narrow linewidth of $17 \mathrm{meV}$ at excitation power density of $3.4 \mathrm{~W} / \mathrm{cm}^{2}$. Temperature-dependent PL spectra follow the Varshni equation well, and peak energy blueshifts by $\sim 45 \mathrm{meV}$ from 300 to $15 \mathrm{~K}$. Power-densitydependent PL spectroscopy manifests direct near-band-edge transition. A low carrier density of 3 $\times 10^{17} \mathrm{~cm}^{-3}$ has been estimated from PL empirical relation, which is close to the critical carrier density of the Mott transition of $2 \times 10^{17} \mathrm{~cm}^{-3}$. (C) 2007 American Institute of Physics.
\end{abstract}

[DOI: $10.1063 / 1.2804568$ ]

Since the revision of InN energy gap to within the infrared (IR) region, InN-based devices are expected to be developed as sensors, solar cells, IR light-emitting diodes, IR laser diodes, and high-frequency electronic devices, among others. ${ }^{1-3}$ However, the fundamental bandgap of InN is still unclear, presumably due to the difficulty in obtaining highquality InN, which largely impedes its device development. As shown in the thin film case, both absorption edge and photoluminescence (PL) peak energy decrease with increasing film thickness and improving film quality. ${ }^{4,5}$ Meanwhile, for one-dimensional nanostructures such as nanowires, nanorods, or nanobelts, despite of their improved structural quality, some anomalous phenomena remain in their optical properties. ${ }^{6-9}$ For instance, negligible changes in peak position of bandgap with increasing temperature, low PL intensity, higher PL peak energy or absorption edge, and broadened linewidth were commonly observed. From fundamental point of view, because the $\mathrm{InN}$ has small effective mass of $0.033 m_{0}$, high free carrier density, and large electron affinity, its Fermi energy is easily located above the conduction band minimum and forms degenerate semiconductor. ${ }^{10-12}$ Hence, the variation in the observed emission wavelength or absorption edge may result from multiple origins, including the quantum confinement and surface electron accumulation effects, the Moss-Burstein shift due to high carrier density, and oxygen incorporation. ${ }^{1,2,6,7,13-16}$

In this letter, quasibulk InN microcrystals were prepared for studying intrinsic properties of InN with barely coupling effects. These microcrystals were grown nearly free from defect and strain. Because the InN microcrystals were di-

\footnotetext{
${ }^{\text {a) }}$ Author to whom correspondence should be addressed; electronic mail:
} chenlc@ntu.edu.tw rectly grown on $\mathrm{Si}$ substrate without $\mathrm{GaN}$ or AlN buffers, no alloys, InGaN or AlInN, could be formed to affect the optical properties. Therefore, the unintentionally doped $\mathrm{InN}$ microcrystals show strong PL emission peak at low temperature with a narrow linewidth of $17 \mathrm{meV}$ at $0.679 \mathrm{eV}$. Temperature-dependent PL spectra follow the Varshni equation well. A very low carrier density of $3 \times 10^{17} \mathrm{~cm}^{-3}$ was estimated, which is close to the nondegenerate semiconductor region. ${ }^{17}$

The growth of InN microcrystals on $\mathrm{Si}(111)$ substrates were performed in a plasma-assisted molecular-beam epitaxy (PAMBE) system. ${ }^{18}$ Details of the growth procedure can be found elsewhere. ${ }^{19}$ Very low-level impurities, such as $\mathrm{H}, \mathrm{O}$, $\mathrm{C}$, etc., were confirmed from an $\mathrm{InN}$ thin film grown by the PAMBE using secondary ion mass spectroscopy analysis. ${ }^{20}$ These microcrystals were grown near the $\mathrm{InN}$ dissociation temperature of $550{ }^{\circ} \mathrm{C}$ under $\mathrm{N}$-rich condition using only one-step growth without any buffer layer. Well-faceted $\mathrm{InN}$ microcrystals grown at $550{ }^{\circ} \mathrm{C}$ are shown in the fieldemission scanning electron microscope (FESEM) image [Fig. 1(a)]. The diameters of the microcrystals were within a range of 0.1-1 $\mu \mathrm{m}$, depending on the growth conditions. In addition, these microcrystals were distributed on the 2 $\times 2 \mathrm{~cm}^{2}$ wafer with the same morphology, as shown in Fig. 1(a). Figure 1(b) shows a transmission electron microscopy (TEM) image taken at the interface between one of the $\mathrm{InN}$ microcrystals and the Si substrate. Neither dislocation nor stacking faults are observed. Moreover, only small strain fields appeared at the interface indicates that the microcrystals were grown under nearly strain-free conditions. Their corresponding selective area electron diffraction (SAED) pattern with zone axes of $\mathrm{InN}$ in $\langle 1 \underline{100\rangle}$ and $\mathrm{Si}$ in $\langle\underline{1} 12\rangle$ orientations, shown in Fig. 1(c), exhibits a growth relation- 

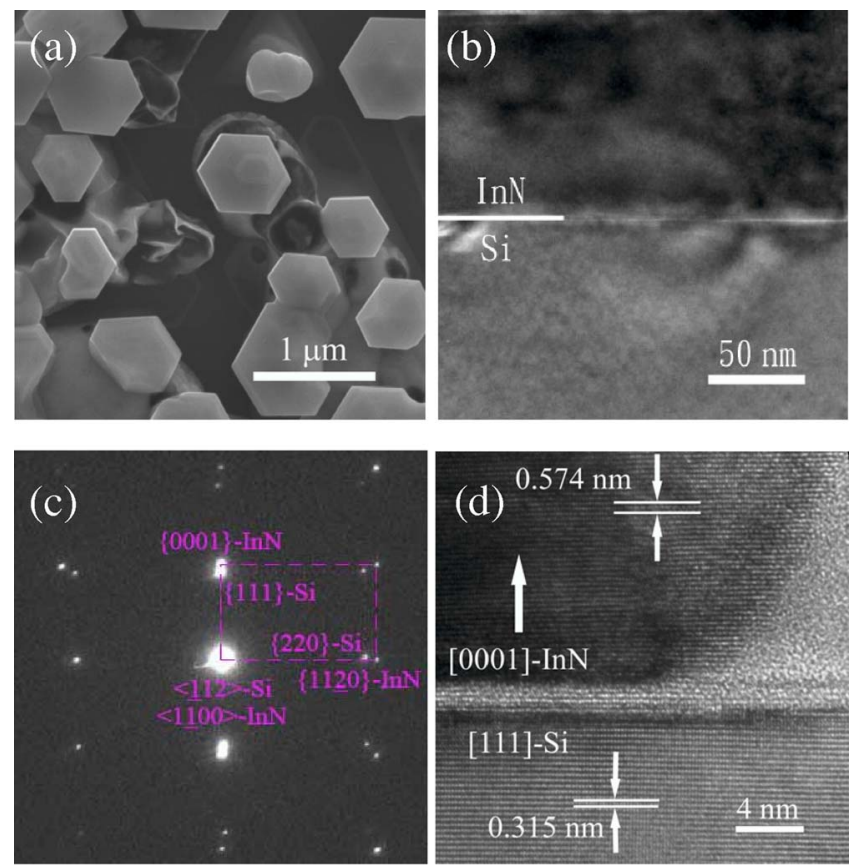

FIG. 1. (Color online) (a) Top-view FESEM image of InN microcrystals. (b) TEM image taken at the interface between Si substrate and an InN microcrystal, and (c) corresponding SAED pattern. (d) high-resolution TEM image taken at the edge of an InN microcrystal.

ship of $\operatorname{InN}(0001) \| \operatorname{Si}(111)$ of the microcrystal. However, not all microcrystals showed the same crystallographic orientation because the large lattice mismatch $(\sim 8 \%)$ between $\mathrm{InN}$ and $\mathrm{Si}$ resulted in three-dimensional (3D) island growth. ${ }^{19}$ In Fig. 1(d), the lattice image reveals the perfection of the microcrystal. Using the interplanar spacing of $d_{\langle 111\rangle}$ $=0.315 \mathrm{~nm}$ of $\mathrm{Si}(111)$ substrate as the reference scale, the lattice constant along the $c$ axis is obtained as $c=0.574 \mathrm{~nm}^{8}$ The point-to-point spatial resolution of the TEM system is $0.17 \mathrm{~nm}$. Additionally, there is an ultrathin amorphous $\mathrm{SiN}_{x}$ layer appeared at the interface due to the reaction of the $\mathrm{Si}$ substrate with active nitrogen. Similar result was also observed when the $\mathrm{GaN}$ was directly deposited on $\mathrm{Si}$ substrate. ${ }^{21}$ The formation of $\mathrm{SiN}_{x}$ layer further promotes the $3 \mathrm{D}$ island growth and results in strain relief of the $\mathrm{InN}$ microcrystals on Si substrate.

PL spectroscopy ${ }^{22}$ was performed to study the optical transitions of the InN microcrystals. Figure 2(a) shows the varied temperature PL spectra. At room temperature, emission peaks were located at 0.633 and $0.639 \mathrm{eV}$ with narrow linewidths of 58 and $64 \mathrm{meV}$ at excitation power densities of 3.4 and $464.0 \mathrm{~W} / \mathrm{cm}^{2}$, respectively. This result is remarkable since it could be comparable with high-quality InN film $(>4 \mu \mathrm{m})$ with a very low carrier density of $2 \times 10^{17} \mathrm{~cm}^{-3}$ and with a narrow PL linewidth of $54 \mathrm{meV}$ at a peak energy of $0.626 \mathrm{eV} .^{5}$ However, when the growth temperature differed largely from the dissociation temperature or when a low-temperature buffer layer was added, the PL peak became broad and shifted toward higher energy, which could be attributed to higher carrier densities. ${ }^{23,24}$ Therefore, it is believed that the effects of structure disorder and of the increase of dislocation extended from the buffer layer generate more carriers in the microcrystals and thin films.

As measurement temperature decreased to $15 \mathrm{~K}$, the PL peak was blueshifted to 0.679 and $0.682 \mathrm{eV}$ with linewidths Downloaded 09 Dec 2008 to 140.112 .113 .225 . Redistribution subject
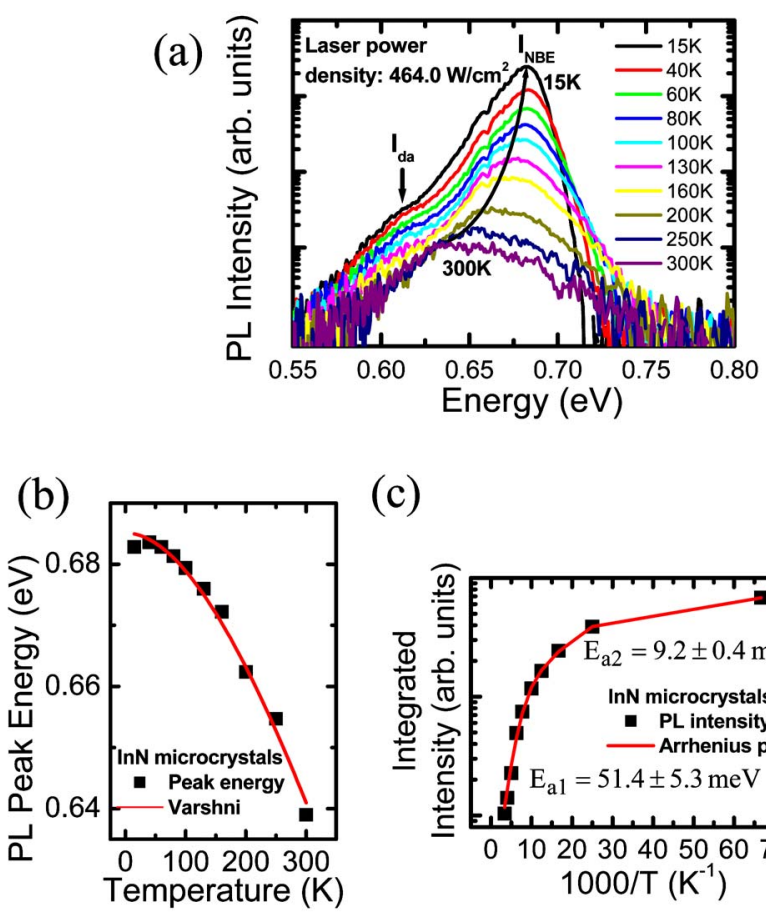

(c)

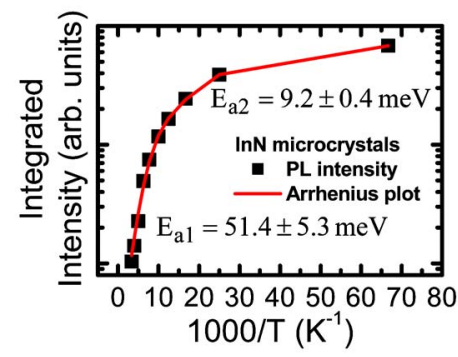

FIG. 2. (Color online) (a) Temperature-dependent PL spectra measured from InN microcrystals. With decreasing temperatures, the $I_{\mathrm{da}}$ emission emerged at the low-energy side of near-band-edge transition. (b) The PL peak energy vs temperature shows a well Varshni's fitting for the experimental data points. (c) Arrhenius plots of the integrated PL intensities for the InN microcrystals.

of 17 and $26 \mathrm{meV}$ at excitation power densities of 3.4 and $464.0 \mathrm{~W} / \mathrm{cm}^{2}$, respectively. The other peak at $0.610 \mathrm{eV}$ appeared at the shoulder of the low-energy side, which has been investigated and identified as $I_{\mathrm{NBE}}$ and $I_{\mathrm{da}}$ transitions, respectively. ${ }^{25,26} I_{\mathrm{NBE}}$ is the near-band-edge transition. $I_{\mathrm{da}}$ transition is attributed to the recombination of degenerate electrons with the holes trapped by deep acceptors, which can be recognized from $I_{\mathrm{NBE}}$ band when the free carrier concentration is in a rather low-density region of $10^{17} \mathrm{~cm}^{-3} \cdot 25,26$ Furthermore, according to the empirical relation between a full width at half maximum of PL emission at low temperature and carrier concentration, ${ }^{23}$ the carrier concentration of InN microcrystals is nearly $3 \times 10^{17} \mathrm{~cm}^{-3}$, which is very close to the critical carrier density of the Mott transition of $2 \times 10^{17} \mathrm{~cm}^{-3} \cdot{ }^{17}$ With increasing temperature, the peak energy of the $I_{\mathrm{NBE}}$ transition shows redshift and finally merges with the $I_{\mathrm{da}}$ transition. This phenomenon is caused by the effect of thermal ionization of electrons and holes in donor and acceptor states, respectively, which results only one broader emission.

Figure 2(b), the plot of PL peak energy versus temperature, shows a result of temperature-induced bandgap shrinkage and is well fitted by the Varshni empirical formula, $E(T)=E(0)-\alpha T^{2} /(T+\beta)$, wherein $T$ is the temperature, $E(0)$ is the peak energy at $0 \mathrm{~K}, \alpha$ is influenced by the thermal expansion and electron-phonon interaction of the fundamental band gap, and $\beta$ is physically associated with the Debye temperature of the crystal. The fitting parameters are $E(0)$ $=685.1 \mathrm{meV}, \alpha=0.46 \mathrm{meV} / \mathrm{K}$, and $\beta=640.3 \mathrm{~K}$. These fitting parameters are reasonably consistent with the low carrier density (mid-10 ${ }^{17} \mathrm{~cm}^{-3}$ ) InN thin film. ${ }^{24}$ Furthermore, the measured PL peak energy blueshifts by $\sim 45 \mathrm{meV}$ with decreasing temperature from 300 to $15 \mathrm{~K}$, and the peak energy to AIP license or copyright; see http://apl.aip.org/apl/copyright.jsp 


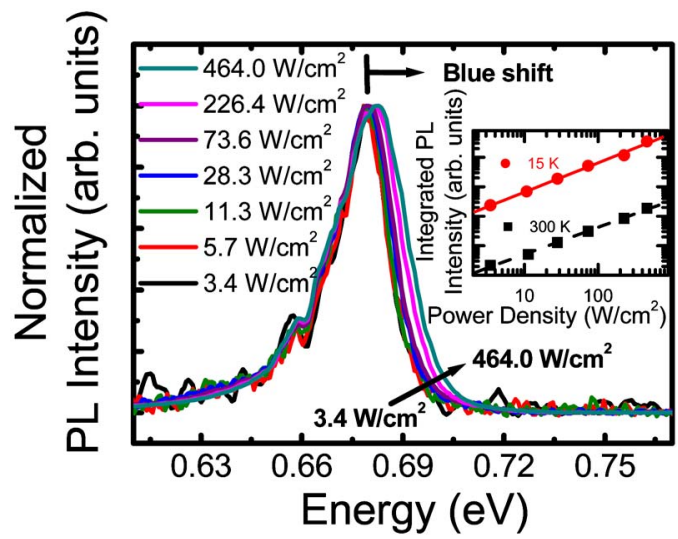

FIG. 3. (Color online) $15 \mathrm{~K}$ variable excitation power densities PL spectra measured from InN microcrystals. The PL intensities were normalized to show a blueshift of peak energy with increasing excitation power density. The inset shows the plot of integrated PL intensity vs excitation power density at temperatures of 15 and $300 \mathrm{~K}$.

at $15 \mathrm{~K}$ is close to the calculated bandgap limit of zero temperature and zero concentration in the range of $0.665-0.670 \mathrm{eV} .^{25}$ Figure 2(c) shows the Arrhenius plot of integrated PL intensities. The thermal activation energies can be determined by the equation, ${ }^{27}$

$$
I=I_{0} /\left(1+A \exp \left(-E_{a 1} / k T\right)+B \exp \left(-E_{a 2} / k T\right)\right),
$$

where $E_{a 1}$ and $E_{a 2}$ are the thermal activation energies at high- and low-temperature regions, respectively, $I_{0}$ is the intensity at low temperature, and the coefficients $A$ and $B$ measure the strengths of both quenching processes. The fitting results of the thermal activation energies is $E_{a 1}$ $=51.4 \pm 5.3 \mathrm{meV}$ in higher temperature range and is $E_{a 2}$ $=9.2 \pm 0.4 \mathrm{meV}$ in lower temperature range. The high thermal activation energy in high temperature range indicates high efficiency of luminescence because of low trapping centers in the microcrystals. The value of $E_{a 2}$ is close to the localization energy originates from the potential fluctuations of randomly located ionized impurities. ${ }^{27}$ These results imply InN microcrystals exhibit low-density localized states from band tail states.

Figure 3 shows spectra of normalized PL intensities with different excitation power densities measured at $15 \mathrm{~K}$. With increasing the excitation power density from 3.4 to $464.0 \mathrm{~W} / \mathrm{cm}^{2}$, the PL peak and linewidth varied from 0.679 to $0.682 \mathrm{eV}$ and 17 to $26 \mathrm{meV}$, respectively, indicating a prominent band filling effect caused by the induced photocarriers. ${ }^{28}$ Furthermore, the logarithmic plot of the integrated PL intensity versus excitation power density yields a slope of 1 at both temperatures of 15 and $300 \mathrm{~K}$ [Inset of Fig. 3], which are consistent with the band-to-band transition in direct bandgap semiconductor. ${ }^{29}$

In conclusion, we have clearly demonstrated strong and narrow PL emission in IR region from nearly defect-free $\mathrm{InN}$ microcrystals. All the PL results show nondegenerate semiconductor behaviors, such as prominent blue shift of bandgap with decreasing temperature and sharp IR PL peak with a very narrow PL linewidth, because of its extremely low intrinsic carrier density and high crystalline quality. The massively reduced number of defects, including dislocations, stacking faults, grain boundaries, and impurities in microc- rystals is an important feature for succeeding $p$-type $\operatorname{InN}$, hence, making future optoelectronic devices possible.

This work was supported by the National Science Council in Taiwan.

${ }^{1}$ V. Y. Davydov, A. A. Klochikhin, R. P. Seisyan, V. V. Emtsev, S. V. Ivanov, F. Bechstedt, J. Furthmüller, H. Harima, A. V. Murdryi, J. Aderhold, O. Semchinova, and J. Graul, Phys. Status Solidi B 229, R1 (2002).

${ }^{2}$ J. Wu, W. Walukiewicz, K. M. Yu, J. W. Arger III, E. E. Haller, H. Lu, W. J. Schaff, Y. Saito, and Y. Nanishi, Appl. Phys. Lett. 80, 3967 (2002).

${ }^{3}$ Y. Nanishi, Y. Saito, and T. Yamaguchi, Jpn. J. Appl. Phys., Part 142 , 2549 (2003).

${ }^{4}$ C. J. Liu, L. A. Bendersky, H. Lu, and W. J. Schaff, Appl. Phys. Lett. 83, 2817 (2003).

${ }^{5}$ G. Koblmüller, C. S. Gallinat, S. Bernardis, J. S. Speck, G. D. Chern, E. D. Readinger, H. Shen, and M. Wraback, Appl. Phys. Lett. 89, 071902 (2006).

${ }^{6}$ C. H. Shen, H. Y. Chen, H. W. Lin, S. Gwo, A. A. Klochikhin, and V. Y. Davydov, Appl. Phys. Lett. 88, 253104 (2006).

${ }^{7}$ T. Stoica, R. J. Meijers, R. Calarco, T. Richter, E. Sutter, and H. Lüth, Nano Lett. 6, 1541 (2006).

${ }^{8}$ M. S. Hu, W. M. Wang, T. T. Chen, L. S. Hong, C. W. Chen, C. C. Chen, Y. F. Chen, K. H. Chen, and L. C. Chen, Adv. Funct. Mater. 16, 537 (2006).

${ }^{9}$ S. C. Shi, C. F. Chen, G. M. Hsu, J. S. Hwang, S. Chattopadhyay, Z. H. Lan, K. H. Chen, and L. C. Chen, Appl. Phys. Lett. 87, 203103 (2005).

${ }^{10}$ Y. M. Chang, H. W. Chu, C. H. Shen, H. Y. Chen, and S. Gwo, Appl. Phys. Lett. 90, 072111 (2007).

${ }^{11}$ E. A. Davis, S. F. J. Cox, R. L. Lichti, and C. G. Van de Walle, Appl. Phys. Lett. 84, 592 (2003).

${ }^{12}$ J. S. Thakur, D. Haddad, V. M. Naik, R. Naik, G. W. Auner, H. Lu, and W. J. Schaff, Phys. Rev. B 71, 115203 (2005).

${ }^{13}$ W. C. Ke, C. P. Fu, C. Y. Chen, L. Lee, C. S. Ku, W. C. Chou, W. H. Chang, M. C. Lee, W. K. Chen, W. J. Lin, and Y. C. Cheng, Appl. Phys. Lett. 88, 191913 (2006)

${ }^{14}$ Z. H. Lan, W. M. Wang, C. L. Sun, S. C. Shi, C. W. Hsu, T. T. Chen, K. H. Chen, C. C. Chen, Y. F. Chen, and L. C. Chen, J. Cryst. Growth 269, 87 (2004).

${ }^{15}$ C. H. Liang, L. C. Chen, J. S. Hwang, K. H. Chen, Y. T. Hung, and Y. F. Chen, Appl. Phys. Lett. 81, 22 (2002).

${ }^{16}$ M. Yoshimoto, H. Yamamoto, W. Huang, H. Harima, J. Saraie, A. Chayahara, and Y. Horino, Appl. Phys. Lett. 83, 3480 (2003).

${ }^{17}$ T. Inushima, M. Haigashiwaki, T. Matsui, T. Takenobu, and M. Motokawa, Phys. Rev. B 72, 085210 (2005).

${ }^{18}$ L. W. Tu, C. L. Hsiao, T. W. Chi, I. Lo, and K. Y. Hsieh, Appl. Phys. Lett. 82, 1601 (2003).

${ }^{19}$ C. L. Hsiao, L. W. Tu, M. Chen, Z. W. Jiang, N. W. Fan, Y. J. Tu, and K. R. Wang, Jpn. J. Appl. Phys., Part 2 44, L1076 (2005).

${ }^{20}$ J. T. Chen, M.S.E. thesis, Tatung University, 2006.

${ }^{21}$ E. Calleja, M. A. Sánchez-García, F. J. Śanchez, F. Calle, F. B. Naranjo, E. Muňoz, S. I. Molina, A. M. Śanchez, F. J. Pacheco, and R. García, J. Cryst. Growth 201/202, 296 (1999).

${ }^{22}$ J. T. Chen, C. L. Hsiao, H. C. Hsu, C. T. Wu, C. L. Yeh, P. C. Wei, L. C. Chen, and K. H. Chen, J. Phys. Chem. A 111, 6755 (2007).

${ }^{23}$ S. P. Fu, T. T. Chen, and Y. F. Chen, Semicond. Sci. Technol. 21, 244 (2006).

${ }^{24}$ J. Wu, W. Walukiewicz, W. Shan, K. M. Yu, J. W. Arger III, E. E. Haller, S. X. Li, E. E. Haller, H. Lu, and W. J. Schaff, J. Appl. Phys. 94, 4457 (2003).

${ }^{25}$ S.-H. Wei, X. Nie, I. G. Batyrev, and S. B. Zhang, Phys. Rev. B 67, 165209 (2003).

${ }^{26}$ A. A. Klochikhin, V. Y. Davydov, V. V. Emtsev, A. V. Sakharov, V. A. Kapitonov, B. A. Andreev, H. Lu, and W. J. Schaff, Phys. Rev. B 71, 195207 (2005).

${ }^{27}$ G. W. Shu, P. F. Wu, M. H. Lo, J. L. Shen, T. Y. Lin, H. J. Chang, Y. F. Chen, C. F. Shih, C. A. Chang, and N. C. Chen, Appl. Phys. Lett. 89, 131913 (2006).

${ }^{28}$ F. Chen, A. N. Cartwright, H. Lu, and W. J. Schaff, Physica E (Amsterdam) 20, 308 (2004).

${ }^{29}$ T. Schmidt, K. Lischka, and W. Zulehner, Phys. Rev. B 45, 8989 (1992). 